\title{
Construction of a risk prediction model for Alzheimer's disease in the elderly population
}

\author{
Lingling Wang ${ }^{1 \dagger}$, Ping $\mathrm{Li}^{2+}$, Ming $\mathrm{Hou}^{2}$, Xiumin Zhang ${ }^{2}$, Xiaolin $\mathrm{CaO}^{2}$ and Hongyan $\mathrm{Li}^{1^{*}}$ (1)
}

\begin{abstract}
Background: Dementia is one of the greatest global health and social care challenges of the twenty-first century. The etiology and pathogenesis of Alzheimer's disease (AD) as the most common type of dementia remain unknown. In this study, a simple nomogram was drawn to predict the risk of AD in the elderly population.

Methods: Nine variables affecting the risk of AD were obtained from 1099 elderly people through clinical data and questionnaires. Least Absolute Shrinkage Selection Operator (LASSO) regression analysis was used to select the best predictor variables, and multivariate logistic regression analysis was used to construct the prediction model. In this study, a graphic tool including 9 predictor variables (nomogram-see precise definition in the text) was drawn to predict the risk of $A D$ in the elderly population. In addition, calibration diagram, receiver operating characteristic (ROC) curve and decision curve analysis (DCA) were used to verify the model.
\end{abstract}

Results: Six predictors namely sex, age, economic status, health status, lifestyle and genetic risk were identified by LASSO regression analysis of nine variables (body mass index, marital status and education level were excluded). The area under the ROC curve in the training set was 0.822 , while that in the validation set was 0.801 , suggesting that the model built with these 6 predictors showed moderate predictive ability. The DCA curve indicated that a nomogram could be applied clinically if the risk threshold was between 30 and $40 \%$ (30 to $42 \%$ in the validation set).

Conclusion: The inclusion of sex, age, economic status, health status, lifestyle and genetic risk into the risk prediction nomogram could improve the ability of the prediction model to predict AD risk in the elderly patients.

Keywords: Alzheimer's disease, Risk factor, Prediction model, Nomogram, Diagnosis

\section{Introduction}

Alzheimer's disease (AD) is a neurodegenerative disease that mainly occurs in the elderly and is the most common cause of dementia [1]. More than $90 \%$ of AD cases occur in people over 65 [2]. With the aging of world population, the prevalence of $\mathrm{AD}$ is on the rise. The

\footnotetext{
* Correspondence: lihongyankhi@163.com

'Lingling Wang and Ping Li contributed equally to this work.

'Department of Neurology, People's Hospital of Xinjiang Uygur Autonomous

Region, NO.91 Tianchi Road, Tianshan District, Urumqi, Xinjiang 830001,

Uygur Autonomous Region, China

Full list of author information is available at the end of the article
}

prevalence of dementia in people aged $\geq 60$ years worldwide is reported to be between 5 and 7\% [3]. Therefore, accurate identification of individuals at high risk of dementia is particularly important for early diagnosis and intervention.

Significant progress has been made in terms of risk factors for AD. For example, numerous studies have shown that risk factors in early years (education), middle age (hypertension, obesity, hearing loss, traumatic brain injury and alcohol abuse) and later years (smoking, depression, physical inactivity, social isolation, diabetes and

C C The Author(s). 2021 Open Access This article is licensed under a Creative Commons Attribution 4.0 International License, which permits use, sharing, adaptation, distribution and reproduction in any medium or format, as long as you give appropriate credit to the original author(s) and the source, provide a link to the Creative Commons licence, and indicate if changes were made. The images or other third party material in this article are included in the article's Creative Commons licence, unless indicated otherwise in a credit line to the material. If material is not included in the article's Creative Commons licence and your intended use is not permitted by statutory regulation or exceeds the permitted use, you will need to obtain permission directly from the copyright holder. To view a copy of this licence, visit http://creativecommons.org/licenses/by/4.0/ The Creative Commons Public Domain Dedication waiver (http://creativecommons.org/publicdomain/zero/1.0/) applies to the data made available in this article, unless otherwise stated in a credit line to the data. 
air pollution) may contribute to an increased risk of dementia [4-6]. Higher levels of childhood education and lifetime education are associated with a lower risk of dementia [7]. Both genetic and lifestyle factors are vital in determining the individual risk of developing $\mathrm{AD}$ and other subtypes of dementia [8]. There is growing evidence that avoiding smoking, physical activity, moderate alcohol consumption and a healthy diet reduce the risk of developing dementia [9-13]. Based on the above factors, we can identify high-risk groups for $\mathrm{AD}$ and carry out targeted disease prevention measures, but there has been no recognized good risk assessment tool.

Multiple studies have demonstrated that nomogram is a novel risk prediction model combining multiple indicators rather than univariate analysis based on multivariate logistic analysis, which is important for screening and clinical practice [14-16]. Nomogram is currently widely used for risk prediction of various diseases, including hypertension [17], stroke [18], etc. The application of the model can accurately screen relevant variables and indicators, and determine the most appropriate risk factors. A previous study [19] constructed a nomogram map to predict the probability of conversion from mild cognitive impairment (MCI) to AD. This study combined neuroimaging features, cerebrospinal fluid (CSF) biomarkers and clinical assessment to play a significant role in clinical diagnosis and prediction. In this study, we constructed a risk prediction model for $\mathrm{AD}$ in the elderly by collecting clinical data and combining with questionnaire data.

\section{Materials and methods Data collection}

Based on a previous research results [20], we finally determined sex, age, body mass index (BMI), marital status, education level, economic status, health status (whether suffering from midlife high blood pressure, diabetes, herpesvirus infection, stroke, traumatic brain injury, depression, etc.), lifestyle (including smoking, exercise, diet, alcohol) and genetic risk (if there is a family history of dementia) as the nine risk factors. A total of 555 medical records of elderly patients with $\mathrm{AD}$ previously diagnosed in our hospital were collected between October 2018 and December 2019, and 544 elderly patients without $\mathrm{AD}$ in this region were investigated. The demographic characteristics including the abovementioned 9 risk factors of all participants were acquired by questionnaire. The study was approved by the Medical Ethics Committee of People's Hospital of Xinjiang Uygur Autonomous Region, and all participants were informed and signed written consent forms.

\section{Inclusion and exclusion criteria}

Inclusion criteria: (1) According to the National Institute on Aging and the Alzheimer's Association (NIA-AA), the diagnostic criteria for $\mathrm{AD}$ were as follows: clinically identified dementia, which was recorded by mini-mental state examination, blessed dementia rating scale, or similar test, and confirmed by a neuropsychological test; deficits in 2 or more domains of cognition; progressive deterioration of memory and other cognitive functions; no disturbance of consciousness; age of onset ranging from 40 to 90 years old, most commonly after 65 years old; no systemic disease or other brain diseases, which could explain the progressive deficits in memory and cognition [21]. (2) Patients were $\geq 60$ years old (since most dementia events occur in the elderly) and had lived in the region for at least 6 months or permanently. Exclusion criteria: (1) Basic information of patients was not available due to cognitive impairment and/or inability to participate independently in the cohort. (2) There were serious organic diseases, such as tumors, major surgery, etc.

\section{Grading criteria}

There are four types of marital status: unmarried, married (first marriage with a spouse, digamy with a spouse, remarriage with a spouse), widowed and divorced. $\mathrm{Pa}$ tients were rated based on current marital status, with 1 representing unmarried, 2 representing widowed or divorced, and 3 representing married with a spouse. The education levels were divided into high (university degree or other professional qualification), middle (high school or junior high school), and low (practical qualification related to work). Economic status was divided into five categories based on the Townsend Deprivation Index (which combines information on social class, employment, cars, housing, etc). Higher scores indicate better marital status, higher education levels and better economic status, respectively.

Health status was evaluated based on current disease information, and diseases such as midlife hypertension, diabetes, herpesvirus infection, stroke, traumatic brain injury and depression were considered comprehensively. The criteria were as follows: one point for having 5 or more diseases, 2 points for having 3 or 4 diseases, 3 points for having 2 or 3 diseases, 4 points for having 1 disease and 5 points for not having any disease. Higher scores represent better health status.

The lifestyle score was based on four established risk factors for dementia (smoking status, physical activity, diet and alcohol consumption). Smoking status was classified as current smoking or non-smoking. Regular physical activity was defined as at least $150 \mathrm{~min}$ of moderate exercise per week or $75 \mathrm{~min}$ of vigorous activity per week. A healthy diet is based on recommendations for cardiometabolic health that focus on eating at least four of seven commonly consumed foods, which are often associated with better later cognition and a reduced risk of 
dementia. Moderate alcohol was defined as 0 to $14 \mathrm{~g} / \mathrm{d}$ for women and 0 to $28 \mathrm{~g} / \mathrm{d}$ for men. Lifestyle scores ranged from 1 to 5 , with a higher score indicating greater adherence to a healthy lifestyle. As for genetic risk, 1 score was for not clear, 2 for no family history of dementia and 3 for family history of dementia.

\section{Statistical analysis}

R 3.6.1 [22] software was used for statistical analysis. First, 1099 participants were randomly divided into a training set (824 participants) and a validation set (275 participants) at a ratio of 3:1 using the $\mathrm{R}$ "caret" package [23]. "glmnet" package [24] was used to run least absolute shrinkage and selection operator (LASSO) regression analysis, which is a contraction and variable selection method for linear regression models. In order to obtain a subset of predictor variables, LASSO regression analysis shrinks the regression coefficient of some variables to zero by imposing constraints on model parameters, thus minimizing the prediction error of quantitative response variables. Variables with zero regression coefficients were excluded from the model after contraction, while variables with non-zero regression coefficients were selected as the most correlated with response variables. We set family = "binomial"', which applies to the binary discrete dependent variable, considering the dependent variable as $\mathrm{AD}$ or not $(0 / 1)$. Then we set type.measure $=$ "deviance", that was $-2 \log$-likelihood. Based on -2log-likelihood and binary discrete dependent variables, LASSO regression analysis in $\mathrm{R}$ software was used to centralize and normalize the contained variables for $\mathrm{k}$-fold (usually 10-fold) crossvalidation, and then the best Lambda value was selected. The model provided by Lambda.lse has good performance, but with the fewest number of independent variables. Therefore, the LASSO method was used to analyze data in the training set to select the best predictors of dementia, including sex, age, BMI, marital status, education level, economic status, health status, lifestyle and genetic risk. The above included variables were used for preliminary screening of risk factor variables.

Then, we used the "rms" package [25] of R language to carry out logistic regression. By introducing the features selected in the LASSO regression model, we used multivariate logistic regression analysis to construct the prediction model. Key features included odds ratios (OR), $95 \%$ confidence intervals (CI), and $p$ values. Statistically significant predictors in both groups were selected to establish the AD risk prediction model and a nomogram prediction model was developed using the rms package of $\mathrm{R}$ language. In addition, several validation methods were used to estimate the accuracy of the risk prediction model by using the data in the training set and the validation set. We used R language "pROC" package [26] for receiver operating characteristic curve (ROC). The area under the curve (AUC) was used to identify the quality of the nomogram to distinguish true positive from false positive. We used the "rms" package to draw and calculate the calibration curve for evaluating the calibration of $\mathrm{AD}$ risk nomogram, accompanied by the HosmerLemeshow test (HLtest.R). The "rmda" package [27] was used for decision curve analysis (DCA) to determine the clinical utility of nomogram in this population based on the net benefit of different threshold probabilities.

\section{Results}

\section{Basic characteristics of participants}

The study included 1099 participants with an average age of $66.85 \pm 4.07$ years, of whom 555 had AD and 544 were non-demented subjects. All participants were randomly divided into the training set $(n=824)$ and the validation set $(n=275)$ at a ratio of $3: 1$. The basic characteristics of all participants were shown in Table 1.

\section{Independent risk factors in the training set}

Multivariate logistic regression analysis showed that sex, age, economic status, health status, lifestyle and genetic risk were risk factors for $\mathrm{AD}$ in the elderly population we studied (Fig. 1).

\section{Prediction model construction}

LASSO regression analysis was used to select the predictor variables from Table 1, and multivariate logistic regression was used to establish the prediction model. Six of the original nine variables were included in the risk prediction model, namely sex, age, economic status, health status, lifestyle and genetic risk. These six variables had non-zero coefficients in the LASSO regression model. The prediction model was represented by a nomogram and it was used for quantitative prediction of the risk probability of developing $\mathrm{AD}$ in the elderly population.

The logistic regression analysis results of these 6 variables were listed in Table 2. Since there were significant statistical differences among these six predictors, they were introduced into the prediction model to develop the $\mathrm{AD}$ risk nomogram (Fig. 2). For example, by using the nomogram model, it could be concluded that a 63year-old man, male, in moderate economic condition and good health, without other diseases, enjoying smoking and drinking, with regular exercises and normal diet, having no genetic risk, had a $33.4 \%$ risk of developing AD.

\section{Prediction model verification}

The ROC curve is used to assess the discriminating ability of the prediction model. For the prediction model, the AUC of the nomogram was 0.822 in the training set 
Table 1 Baseline characteristics of all participants

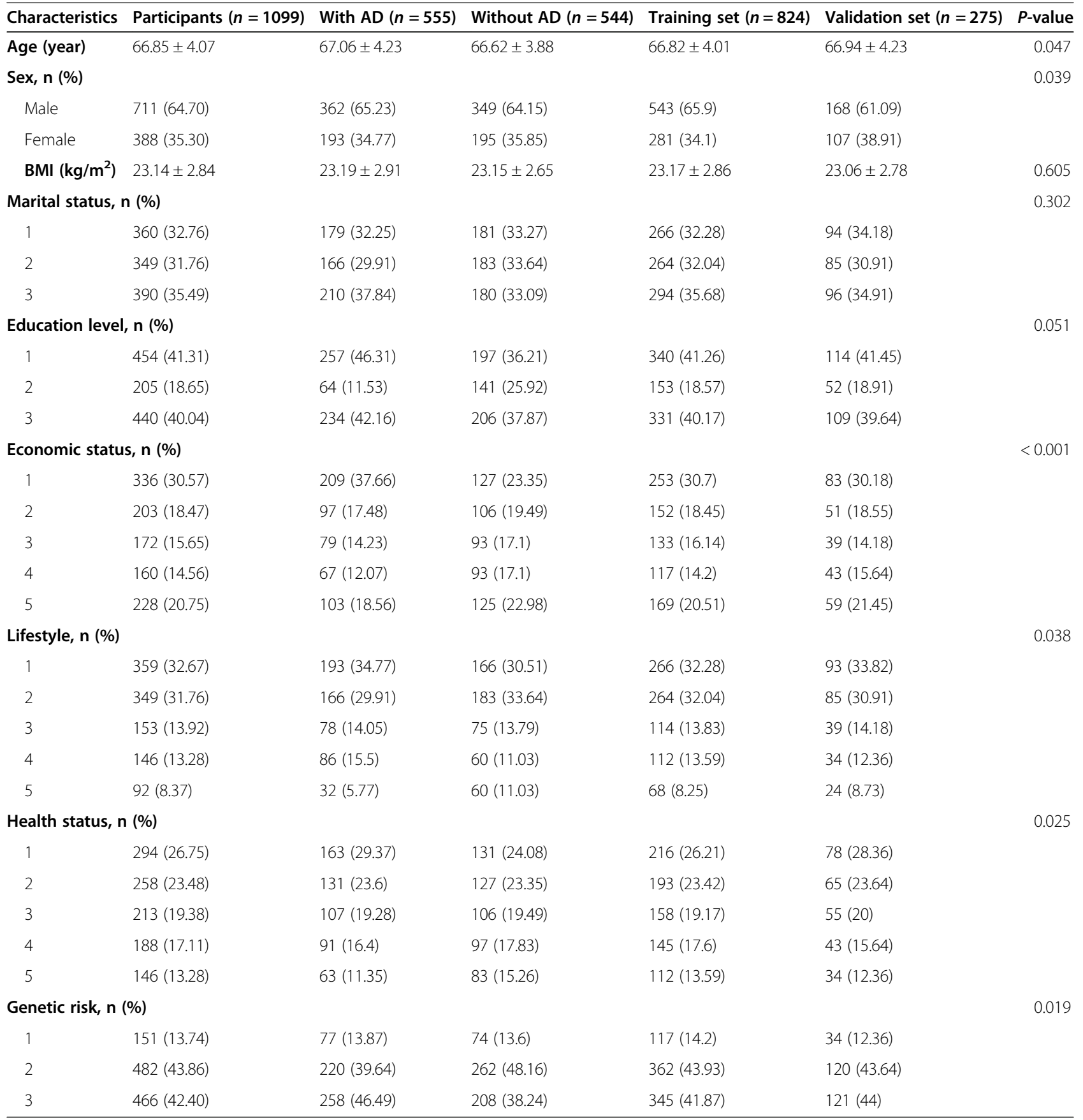

and 0.801 in the validation set (Fig. 3), indicating good performance of the model.

Calibration chart and Hosmer-Lemeshow test were used to calibrate the prediction model. It could be seen from the calibration curve that the prediction model had a good fit with the validation set. Hosmer-Lemeshow test demonstrated that the predicted probability was highly consistent with the actual probability (training set, $p=0.997$; validation set, $p=0.994$ ) (Fig. 4).
DCA results exhibited that the threshold probabilities of training set and validation set in the prediction model were $30-40 \%$ and $30-42 \%$, respectively (Fig. 5), indicating that the model had good application value.

\section{Discussion}

In this study, we constructed a risk prediction model for $\mathrm{AD}$ in the elderly. Sex, age, economic status, health status, lifestyle and genetic risk are independent risk factors for $\mathrm{AD}$ in the elderly. Age is our first consideration. 


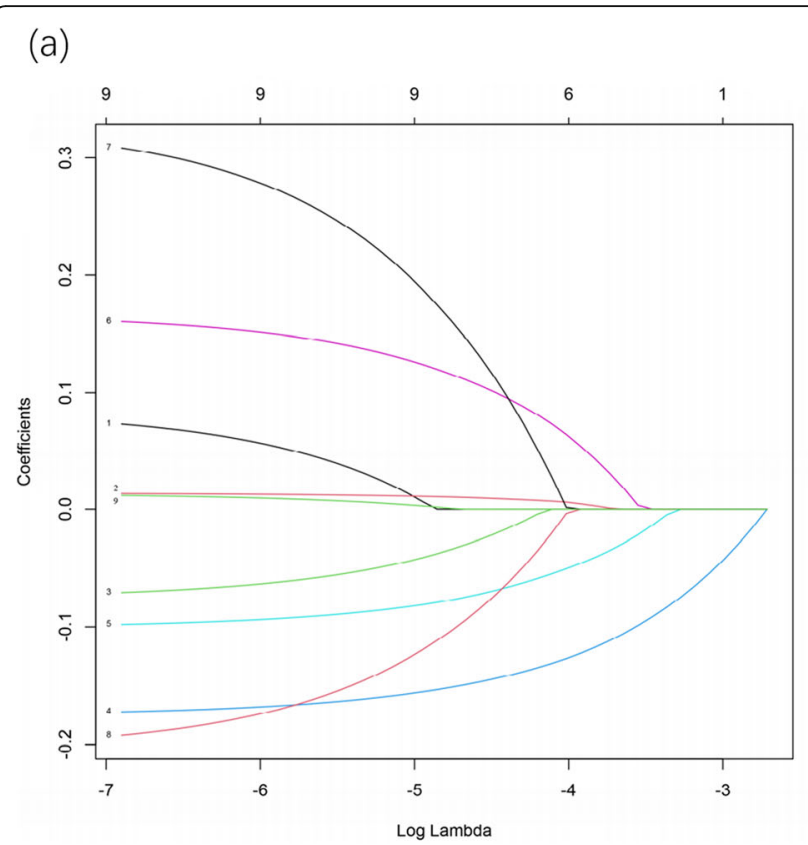

(b)

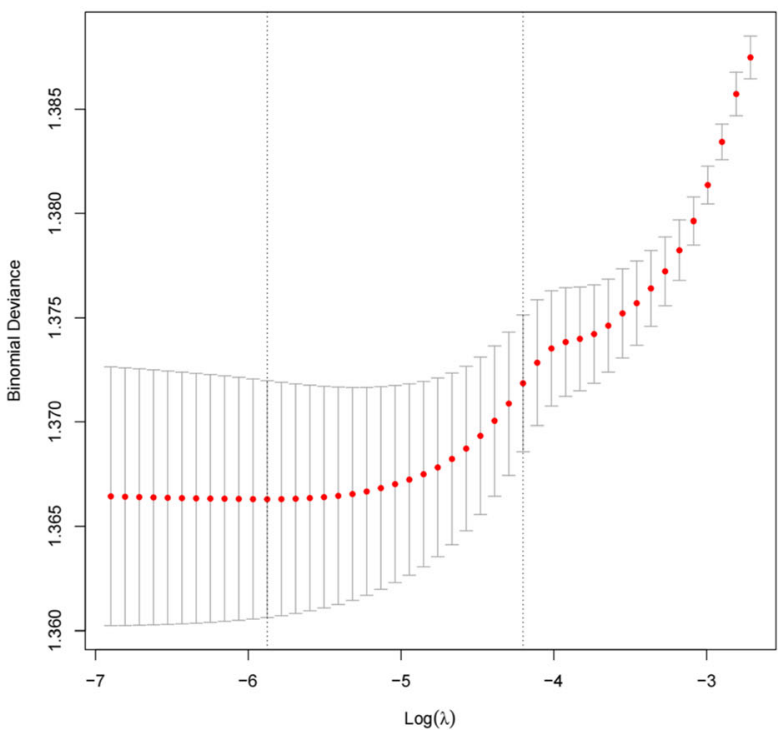

Fig. 1 Selection of variables by LASSO binary logistic regression model and construction of coefficient distribution map according to log (lambda) sequence. a By deducing the best lambda, six variables with non-zero coefficients were selected; $\mathbf{b}$ After verifying the best parameter (lambda) in the LASSO model, a partial likelihood deviance (binomial deviance) curve was plotted versus log (lambda), and a vertical dotted line was plotted with 1 standard error

Since the majority of AD onset occurs over 60 years old, our study was also targeted at the elderly population aged 260. Age is an important risk factor for developing AD. Older age indicates higher risk of developing $\mathrm{AD}$, and age has the greatest impact on advanced dementia compared to other factors [28]. A study suggests that sex difference is another important factor for $\mathrm{AD}$, which may involve the secretion of female hormones [29]. The latest report shows that the bone cell-derived hormone osteocalcin (OCN) plays a key role in cognition [30]. OCN levels are associated with bone density and bone conversion, and therefore are highly affected by changes associated with menopause, increasing risk of disease in menopausal women [30]. All of these studies suggest that women are at greater risk of developing dementia in old age, which is consistent with our risk prediction model.
Previous epidemiological studies on lifestyle and dementia have considered diet [31], physical activity [32] and participation in cognitive activities [33] as risk factors. Another two prospective cohort studies of the elderly have linked a healthy lifestyle with a reduced risk of AD [34]. Specifically, the risk of developing AD of the elderly who also adhere to four or five healthy behaviors (high-quality diet, participation in cognitive activities, regular physical activity, light to moderate alcohol and non-smoking) is $60 \%$ lower compared with that of people who have none or only one healthy behavior. In this study, participants were rated on their adherence to a healthy lifestyle to predict their risk of disease. In addition, the patient's own health is also an important factor to be considered. Multiple studies have shown that hypertension increases the risk of cognitive

Table 2 Logistic regression analysis of risk predictors for AD in the elderly

\begin{tabular}{|c|c|c|c|c|c|}
\hline Intercept and variables & coefficient & $Z$ value & $P$-value & Odds Ratio & $95 \mathrm{Cl} \%$ \\
\hline Intercept & -0.8911 & -0.82 & & & \\
\hline Sex & 0.033 & 0.26 & 0.023 & 1.034 & $0.804-1.329$ \\
\hline Age & 0.0205 & 1.34 & 0.019 & 1.086 & $0.963-1.224$ \\
\hline Economic & -0.1756 & -4.35 & 0.012 & 0.591 & $0.466-0.749$ \\
\hline Lifestyle & -0.0988 & -2.21 & 0.034 & 0.743 & $0.572-0.967$ \\
\hline Heredity & 0.1702 & 1.92 & 0.004 & 1.405 & $0.993-1.989$ \\
\hline Health & -0.0525 & -1.09 & 0.001 & 0.901 & $0.745-1.088$ \\
\hline
\end{tabular}




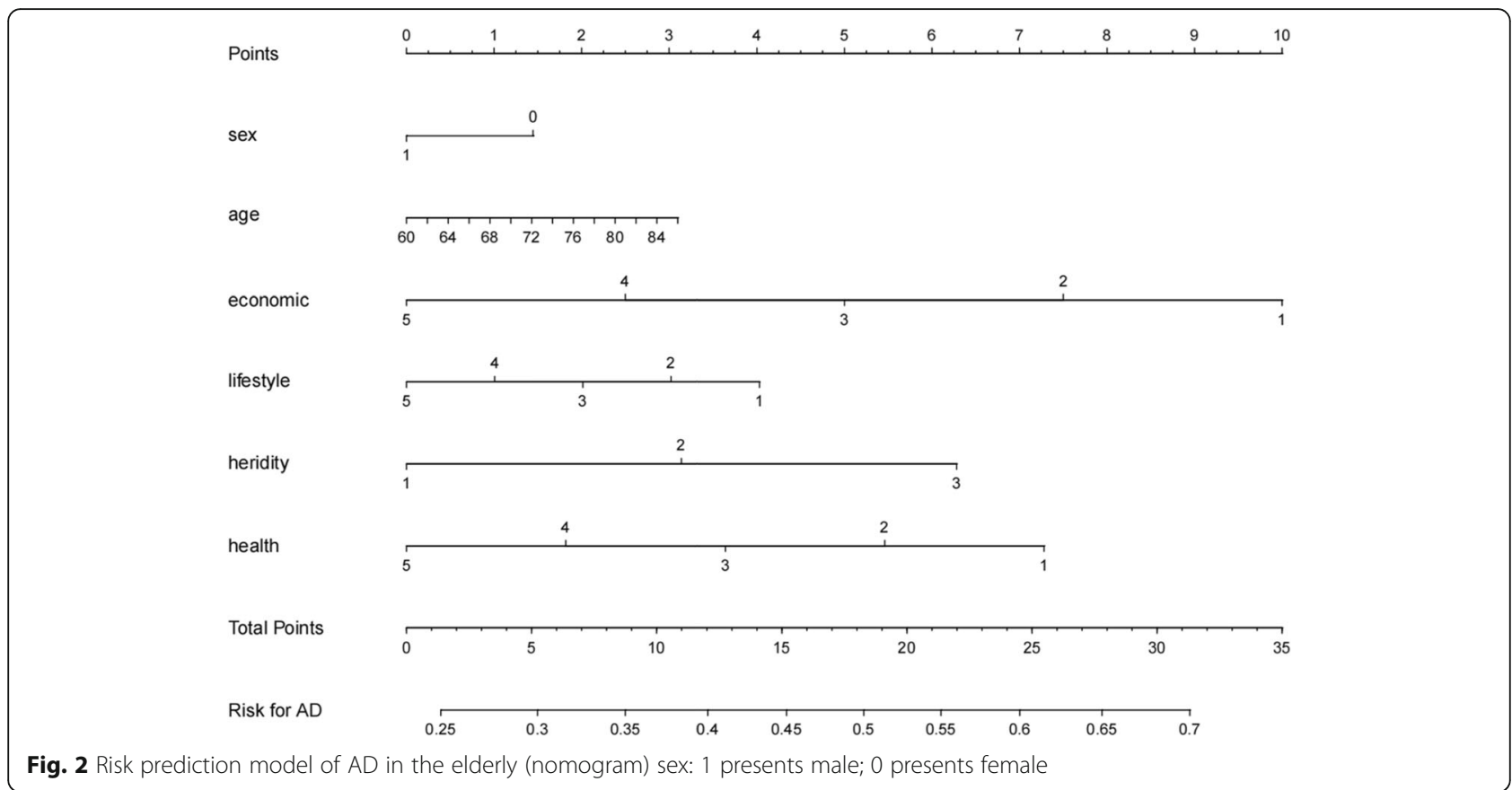

impairment [35] and stroke, of which stroke has been identified as an independent risk factor for dementia [36]. Similarly, elevated glucose can decrease cognitive function and increase the risk of $\mathrm{AD}$ [37]. Our study focused on predicting the risk of $\mathrm{AD}$ by targeting midlife hypertension, diabetes, herpesvirus infection, stroke, traumatic brain injury, and depression. Notably, a number of studies have also shown a link between adverse childhood experiences, psychiatric symptoms and dementia. A large cohort study found that older Japanese who had three or more adverse childhood experiences had an increased risk of dementia [38]. Another study (a)

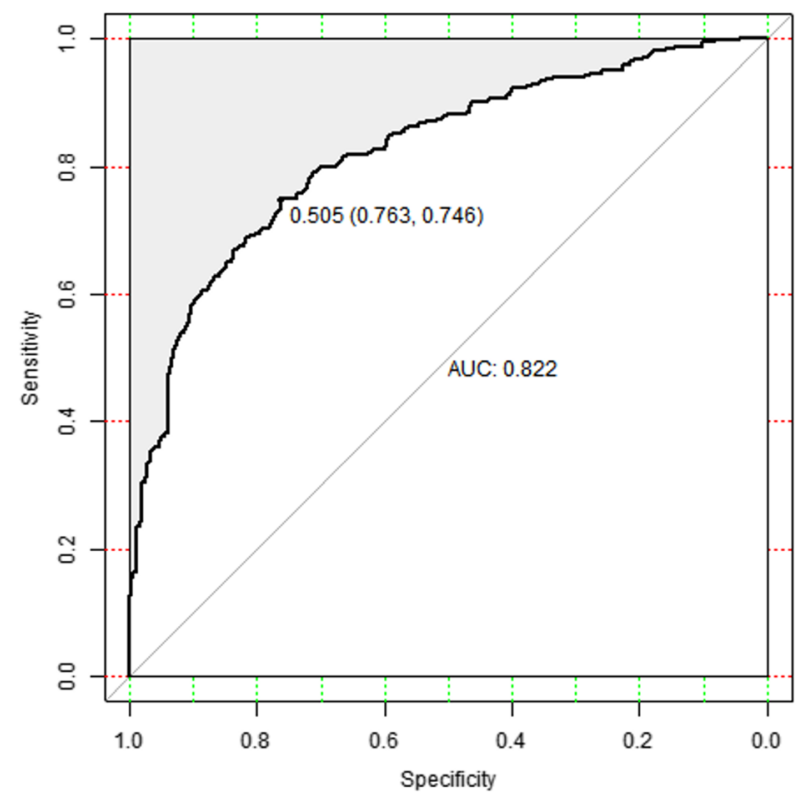

(b)

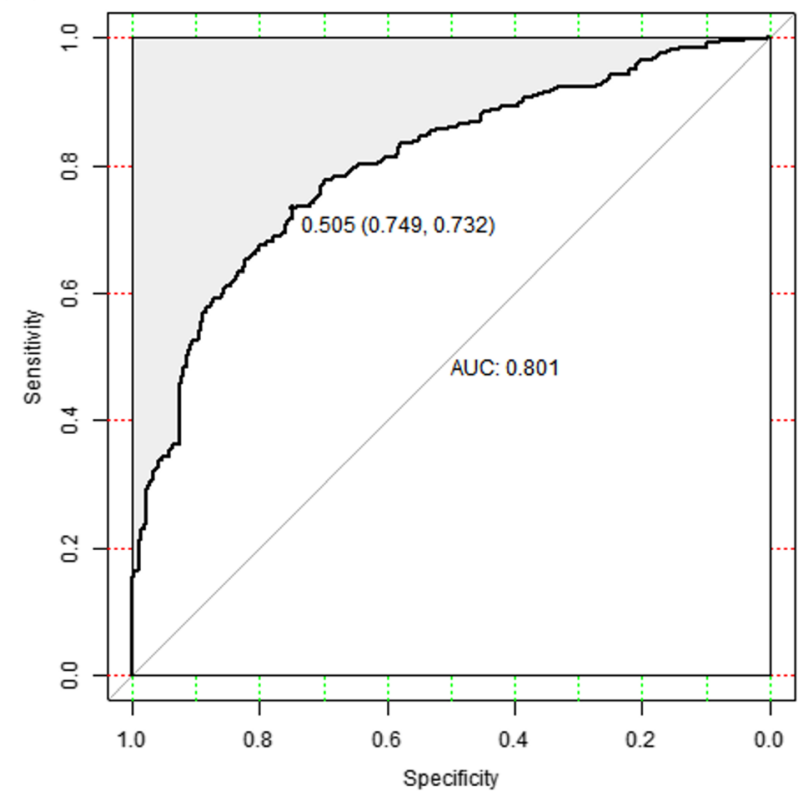

Fig. 3 ROC curve validation of risk prediction nomogram for AD in the elderly. a Training set: optimal threshold: 0.505; corresponding specificity and sensitivity: $0.763,0.746$; $\mathbf{b}$ Validation set: optimal threshold: 0.505; corresponding specificity and sensitivity: $0.749,0.732$. The black bold line represents the performance of the nomogram in the training set and validation set. The $y$-axis represents the true positive rate of risk prediction, and the $x$-axis represents the false positive rate of risk prediction 
(a)

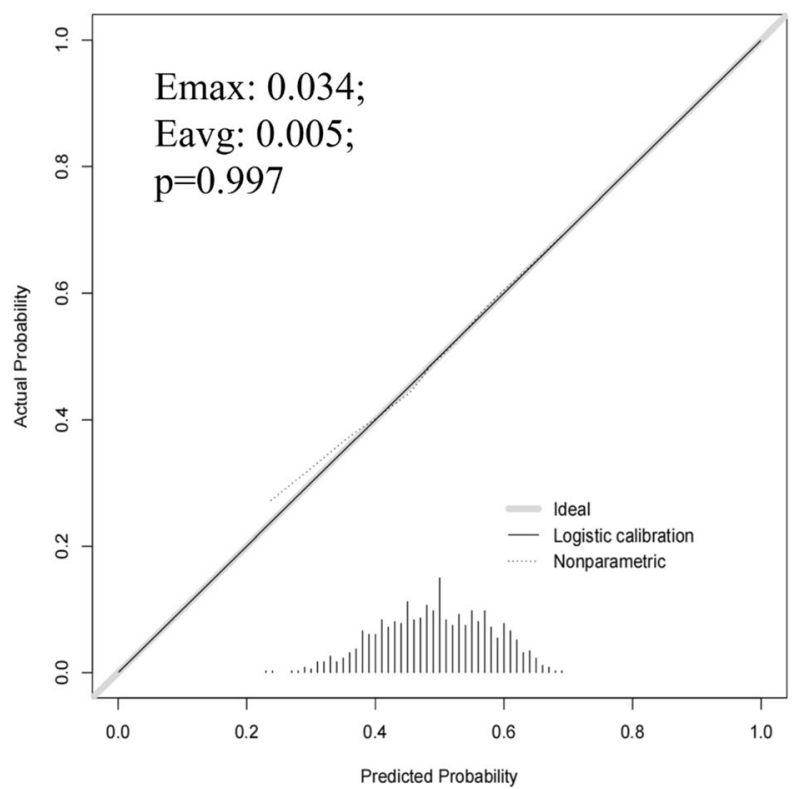

(b)

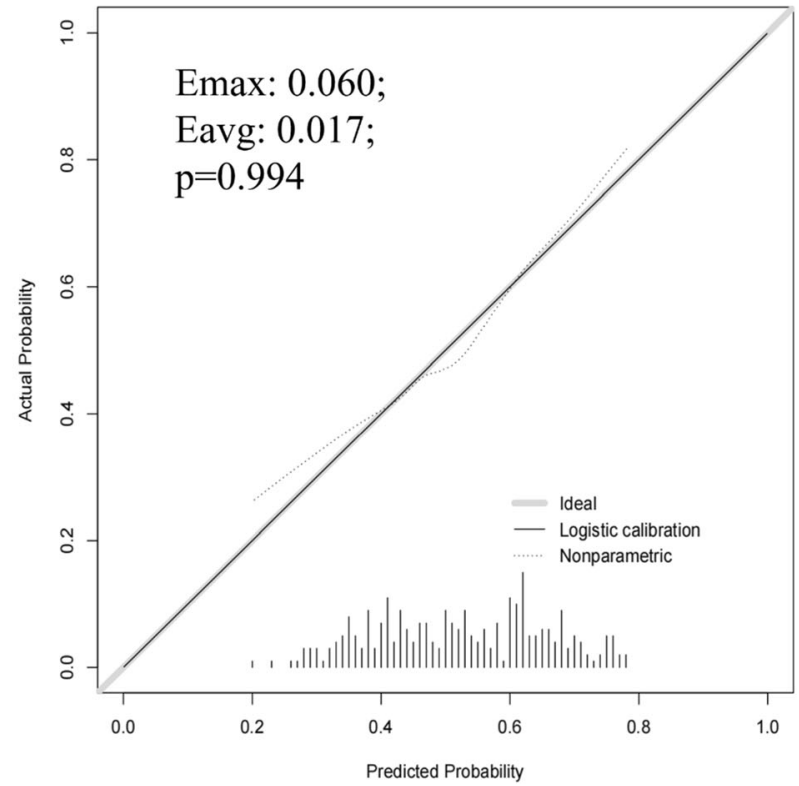

Fig. 4 Calibration curve of risk prediction for AD in the elderly. a Training set; $\mathbf{b}$ Validation set. Emax: the maximum offset between the model and the ideal model; Eavg: the minimal offset between the model and the ideal model. $p>0.05$ indicates passing the calibration test. The black solid line above the $x$-axis represents sample distribution. The dotted lines on the diagonal represent the perfect prediction of the ideal model, and the solid lines represent the performance of the training set and the validation set. The closer the solid line is to the dotted line, the better the predictive effect. The $y$-axis represents the actual diagnosed cases of AD, and the $x$-axis represents the predicted risk of AD

suggested that chronic psychosocial stress may exacerbate synaptic dysfunction and cognitive impairment in AD through stress-induced abnormalities in microglial function [39]. In addition, some studies found that symptoms such as anxiety and apathy also increase the risk of $\mathrm{AD}$ [40-42]. These factors were ignored in our study, which may have led our model to underestimate the risk of $\mathrm{AD}$.

The risk of $\mathrm{AD}$ is associated with a variety of genes, and the APOE on chromosome 19 was the first gene identified to be associated with late-onset $\mathrm{AD}$. Up to now, more than 50 risk gene loci have been screened by using genome-wide association technology, and 11 significant load susceptibility loci have been found, and the potential pathogenic mechanism of $\mathrm{AD}$ has been explained in terms of cell pathway, immune response, somatic mutation, epigenetics and other aspects [43]. This study evaluated genetic risk based on the family history of dementia. Remarkably, the economic status of all participants was also taken as a predictor in this study. Several studies have shown a strong link between socioeconomic status in early life and the risk of dementia later, with low socioeconomic status often associated with increased morbidity and mortality [44]. The reason might be that low-income population have less access to health care and engage in unhealthy behaviors (such as smoking, an unhealthy diet, alcohol abuse and lack of exercises) more often.
Based on the results of the above risk factors, it is necessary to develop more models to better identify people with risk of $\mathrm{AD}$. An example is that five potential risk factors for $\mathrm{AD}$ were identified by using an extended method of Mendelian randomization (MR) - multivariate MR (MVMR) and MR based on Bayesian model averaging (MR-BMA) [45]. Such high-throughput trials can more accurately reflect risk factors for the disease. Another study found that the Framingham cardiovascular Risk Score (FRS) had significant application in predicting dementia risk, particularly the effects of factors such as age and cardiometabolism [46]. In contrast, we applied the nomogram to $\mathrm{AD}$ risk prediction. The risk prediction model is of great value in clinical research due to its convenience in application and high diagnostic performance.

This study still has some limitations. First of all, due to limited funds and manpower, we failed to detect the genetic genes and biochemical indicators of the population. Second, the indicators we eventually included were a broad category that could be subdivided according to existing research results. For example, the intake of deep-sea fish, vegetables and fruits has a large proportion in the diet, and periodontitis, hearing impairment and sleep disorder are also important factors to evaluate the health status. Finally, it is necessary to expand the scope of the study population, including the number of subjects and their region, to improve our model. 
(a)

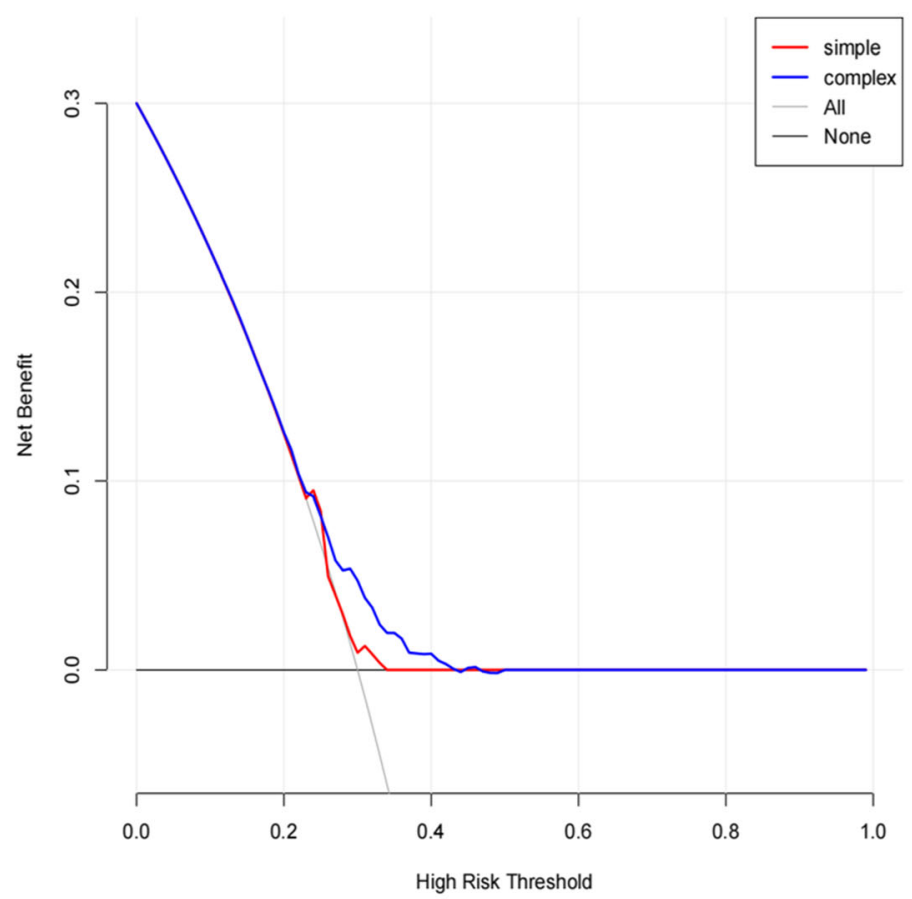

(b)

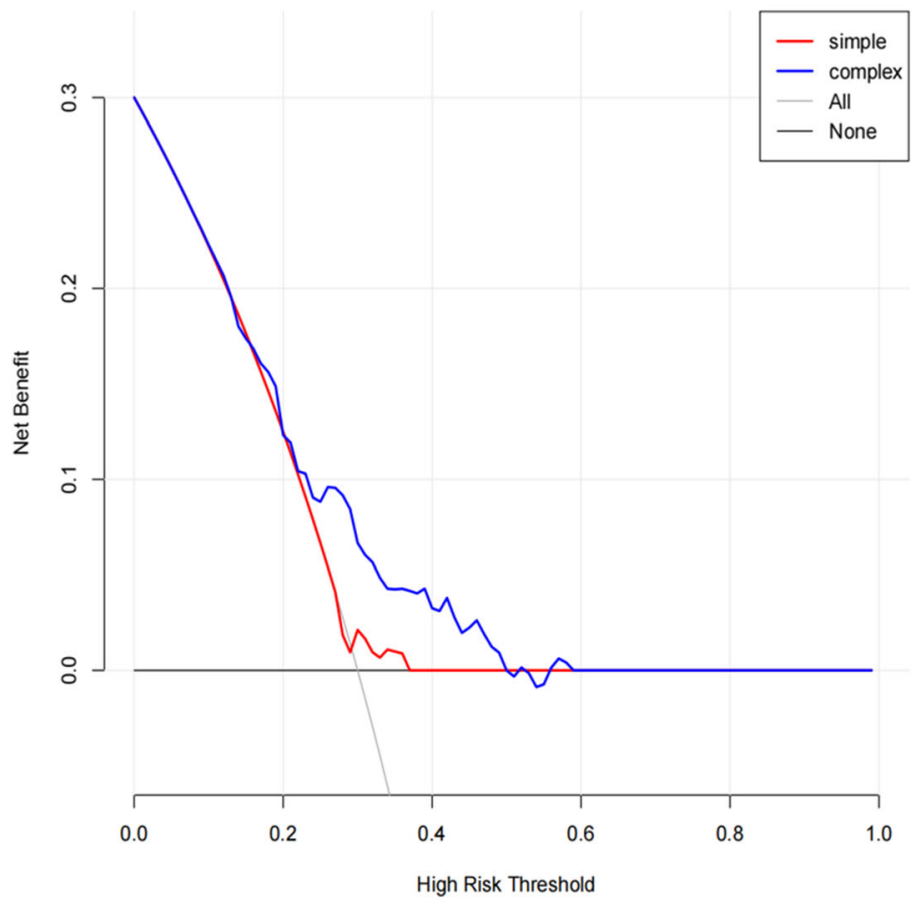

Fig. 5 DCA of risk prediction nomogram for AD in the elderly. a Training set; $\mathbf{b}$ Validation set. The black solid line represents the assumption that none of the participants have AD, and gray solid line represents the assumption that all of the participants have AD. The blue thick solid line represents the composited model, combined with sex, age, economic status, health status, lifestyle and genetic risk as prediction methods, and developing $A D$ as the result. The red thick solid line represents a simple model with only a single risk factor included. The $y$-axis is net benefit, and the $x$-axis is threshold probability 
To sum up, this study investigated the risk factors for $\mathrm{AD}$ in the elderly population, and used the nomogram to construct a model to predict the risk of AD via sex, age, economic status, health status, lifestyle and genetic risk. These risk factors are of great significance for early screening and timely prevention of AD. People can significantly reduce the risk of $\mathrm{AD}$ by adopting a healthy lifestyle, such as not smoking, drinking as little as possible or not drinking, having a healthy diet, exercising more, and early treatment of various diseases (diabetes, hypertension, anxiety, depression, etc.). In addition, the indicators of this model are relatively easy to acquire and include major risk factors, which can be widely applied to the risk prediction of AD in the elderly population. Based on the assessment, corresponding measures can be taken to reduce the risk of the disease.

\section{Abbreviations}

AD: Alzheimer's disease; LASSO: Least Absolute Shrinkage Selection Operator; ROC: Receiver operating characteristic; DCA: Decision curve analysis; MCl: Mild cognitive impairment; CSF: Cerebrospinal fluid; BMI: Body mass index; NIA-AA: National Institute on Aging and the Alzheimer's Association; AUC: Area under the curve; HLtest.R: Hosmer-Lemeshow test:

OCN: Osteocalcin; MR: Mendelian randomization; MVMR: Multivariate MR; MRBMA: MR based on Bayesian model averaging; FRS: Framingham cardiovascular Risk Score

\section{Supplementary Information}

The online version contains supplementary material available at https://doi. org/10.1186/s12883-021-02276-8.

Additional file 1.

Additional file 2.

\section{Acknowledgements}

Not Applicable.

\section{Authors' contributions}

$\mathrm{MH}$ and LLW contributed to the study design. XMZ conducted the literature search. XLC acquired the data. LLW wrote the article. PL performed data analysis and drafted and revised the article. HYL gave the final approval of the version to be submitted. The authors read and approved the final manuscript.

\section{Funding}

This study was supported by the funds from youth research fund of Nursing College of Xinjiang Medical University (HLQN-2019-19) which contributed to the design of the study and collection.

\section{Availability of data and materials}

The datasets used and/or analyzed during the current study are available from the corresponding author on reasonable request.

\section{Declarations}

Ethics approval and consent to participate

Not Applicable.

\section{Consent for publication}

Not Applicable.

\section{Competing interests}

The authors declare that they have no potential conflicts of interest.

\section{Author details}

'Department of Neurology, People's Hospital of Xinjiang Uygur Autonomous Region, NO.91 Tianchi Road, Tianshan District, Urumqi, Xinjiang 830001, Uygur Autonomous Region, China. ${ }^{2}$ Department of Nursing, People's Hospital of Xinjiang Uygur Autonomous Region, Uygur Autonomous Region, Xinjiang 830001, China.

Received: 10 January 2021 Accepted: 9 June 2021

Published online: 07 July 2021

\section{References}

1. Winblad B, Amouyel P, Andrieu S, Ballard C, Brayne C, Brodaty H, et al. Defeating Alzheimer's disease and other dementias: a priority for European science and society. Lancet Neurol. 2016;15(5):455-532. https://doi.org/10.1 016/S1474-4422(16)00062-4.

2. Reilly S, Miranda-Castillo C, Malouf R, Hoe J, Toot S, Challis D, et al. Case management approaches to home support for people with dementia. Cochrane Database Syst Rev. 2015;1(1):Cd008345.

3. Prince $M$, Bryce $R$, Albanese $E$, Wimo A, Ribeiro W, Ferri CP. The global prevalence of dementia: a systematic review and metaanalysis. Alzheimer's Dementia. 2013;9(1):63-75 e62.

4. Livingston G, Huntley J, Sommerlad A, Ames D, Ballard C, Banerjee S, et al. Dementia prevention, intervention, and care: 2020 report of the Lancet Commission. Lancet. 2020;396(10248):413-46.

5. Mortamais M, Gutierrez LA, de Hoogh K, Chen J, Vienneau D, Carrière I, et al Long-term exposure to ambient air pollution and risk of dementia: results of the prospective Three-City study. Environ Int. 2021;148:106376. https:// doi.org/10.1016/j.envint.2020.106376.

6. Bloomberg M, Dugravot A, Dumurgier J, Kivimaki M, Fayosse A, Steptoe A, et al. Sex differences and the role of education in cognitive ageing: analysis of two UK-based prospective cohort studies. Lancet Public Health. 2021;6(2): e106-15. https://doi.org/10.1016/S2468-2667(20)30258-9.

7. Larsson SC, Traylor M, Malik R, Dichgans M, Burgess S, Markus HS. Modifiable pathways in Alzheimer's disease: Mendelian randomisation analysis. BMJ. 2017;359:55375.

8. Mangialasche F, Kivipelto M, Solomon A, Fratiglioni L. Dementia prevention: current epidemiological evidence and future perspective. Alzheimers Res Ther. 2012;4(1):6. https://doi.org/10.1186/alzrt104.

9. Zhong G, Wang Y, Zhang Y, Guo JJ, Zhao Y. Smoking is associated with an increased risk of dementia: a meta-analysis of prospective cohort studies with investigation of potential effect modifiers. PLoS One. 2015;10(3): e0118333. https://doi.org/10.1371/journal.pone.0118333.

10. Yu F, Vock DM, Zhang L, Salisbury D, Nelson NW, Chow LS, et al. Cognitive effects of aerobic exercise in Alzheimer's disease: a pilot randomized controlled trial. J Alzheimer's Dis. 2021;80(1):233-44. https://doi.org/10.3233/ JAD-201100.

11. Xu W, Wang H, Wan Y, Tan C, Li J, Tan L, et al. Alcohol consumption and dementia risk: a dose-response meta-analysis of prospective studies. Eur J Epidemiol. 2017;32(1):31-42. https://doi.org/10.1007/s10654-017-0225-3.

12. Cremonini AL, Caffa I, Cea M, Nencioni A, Odetti $P$, Monacelli F. Nutrients in the prevention of Alzheimer's disease. Oxidative Med Cell Longev. 2019; 2019:9874159.

13. Wesselman LMP, van Lent DM, Schröder A, van de Rest O, Peters O, Menne $F$, et al. Dietary patterns are related to cognitive functioning in elderly enriched with individuals at increased risk for Alzheimer's disease. Eur J Nutr. 2021;60(2):849-60. https://doi.org/10.1007/s00394-020-02257-6.

14. Han K, Yun JS, Park YM, Ahn YB, Cho JH, Cha SA, et al. Development and validation of a risk prediction model for severe hypoglycemia in adult patients with type 2 diabetes: a nationwide population-based cohort study. Clin Epidemiol. 2018;10:1545-59. https://doi.org/10.2147/CLEP.S169835.

15. Kim SY, Cho N, Choi Y, Lee SH, Ha SM, Kim ES, et al. Factors affecting pathologic complete response following neoadjuvant chemotherapy in breast Cancer: development and validation of a predictive nomogram. Radiology. 2021;299(2):290-300. https://doi.org/10.1148/radiol.2021203871.

16. Pan M, Yang Y, Teng T, Lu F, Chen Y, Huang H. Development and validation of a simple-to-use nomogram to predict liver metastasis in patients with pancreatic neuroendocrine neoplasms: a large cohort study. BMC Gastroenterol. 2021;21(1):101. https://doi.org/10.1186/s12876-021-01685-w.

17. Abraldes JG, Bureau C, Stefanescu H, Augustin S, Ney M, Blasco H, et al. Noninvasive tools and risk of clinically significant portal hypertension and 
varices in compensated cirrhosis: The "Anticipate" study. Hepatology. 2016; 64(6):2173-84.

18. Cappellari M, Turcato G, Forlivesi S, Zivelonghi C, Bovi P, Bonetti B, et al. STARTING-SICH nomogram to predict symptomatic intracerebral hemorrhage after intravenous thrombolysis for stroke. Stroke. 2018:49(2): 397-404. https://doi.org/10.1161/STROKEAHA.117.018427.

19. Huang K, Lin Y, Yang L, Wang Y, Cai S, Pang L, et al. A multipredictor model to predict the conversion of mild cognitive impairment to Alzheimer's disease by using a predictive nomogram. Neuropsychopharmacology. 2020; 45(2):358-66. https://doi.org/10.1038/s41386-019-0551-0.

20. Lourida I, Hannon E, Littlejohns TJ, Langa KM, Hyppönen E, Kuzma E, et al. Association of Lifestyle and Genetic Risk with Incidence of dementia. Jama. 2019;322(5):430-7. https://doi.org/10.1001/jama.2019.9879.

21. McKhann G, Drachman D, Folstein M, Katzman R, Price D, Stadlan EM. Clinical diagnosis of Alzheimer's disease: report of the NINCDS-ADRDA work group under the auspices of Department of Health and Human Services Task Force on Alzheimer's disease. Neurology. 1984;34(7):939-44. https://doi. org/10.1212/WNL.34.7.939.

22. Team RC. R: A language and environment for statistical computing. R Foundation for Statistical Computing, Vienna, Austria; 2021.

23. Kuhn M. Caret: Classification and regression training; 2013. p. 1.

24. Friedman J, Hastie T, Tibshirani R. Regularization paths for generalized linear models via coordinate descent. J Stat Softw. 2010;33(1):1-22.

25. Harrell FEJ. rms: Regression Modeling Strategies. R package version 6.1-1. 2021. Available online: https://CRAN.Rproject.org/package=rms.

26. Robin X, Turck N, Hainard A, Tiberti N, Lisacek F, Sanchez JC, et al. pROC: an open-source package for $\mathrm{R}$ and $\mathrm{S}+$ to analyze and compare ROC curves. BMC Bioinformatics. 2011;12(1):77. https://doi.org/10.1186/1471-2105-12-77.

27. Brown M. rmda: Risk Model Decision Analysis; 2017.

28. Masters CL. Major risk factors for Alzheimer's disease: age and genetics. Lancet Neurol. 2020;19(6):475-6. https://doi.org/10.1016/S1474-4422(20)301 55-1.

29. Jiang J, Young K, Pike CJ. Second to fourth digit ratio $(2 \mathrm{D}, 4 \mathrm{D})$ is associated with dementia in women. Early Hum Dev. 2020;149:105152. https://doi. org/10.1016/j.earlhumdev.2020.105152.

30. Schatz M, Saravanan S, d'Adesky ND, Bramlett H, Perez-Pinzon MA, Raval AP. Osteocalcin, ovarian senescence, and brain health. Front Neuroendocrinol. 2020:59:100861. https://doi.org/10.1016/i.yfrne.2020.100861.

31. Morris MC, Tangney CC, Wang Y, Sacks FM, Bennett DA, Aggarwal NT. MIND diet associated with reduced incidence of Alzheimer's disease. Alzheimer's Dementia. 2015;11(9):1007-14. https://doi.org/10.1016/j.jalz.2014.11.009.

32. Hamer M, Chida Y. Physical activity and risk of neurodegenerative disease: a systematic review of prospective evidence. Psychol Med. 2009;39(1):3-11. https://doi.org/10.1017/S0033291708003681.

33. Wilson RS, Segawa E, Boyle PA, Bennett DA. Influence of late-life cognitive activity on cognitive health. Neurology. 2012;78(15):1123-9. https://doi.org/1 0.1212 WNL.0b013e31824f8c03.

34. Dhana K, Evans DA, Rajan KB, Bennett DA, Morris MC. Healthy lifestyle and the risk of Alzheimer dementia: findings from 2 longitudinal studies. Neurology. 2020;95(4):e374-83. https://doi.org/10.1212/WNL. 0000000000009816.

35. Power MC, Weuve J, Gagne JJ, McQueen MB, Viswanathan A, Blacker D. The association between blood pressure and incident Alzheimer disease: a systematic review and meta-analysis. Epidemiology. 2011;22(5):646-59.

36. Dregan A, Wolfe CD, Gulliford MC. Does the influence of stroke on dementia vary by different levels of prestroke cognitive functioning?: a cohort study. Stroke. 2013;44(12):3445-51. https://doi.org/10.1161/STROKEA HA.113.002990.

37. Sun Y, Ma C, Sun H, Wang H, Peng W, Zhou Z, et al. Metabolism: a nove shared link between diabetes mellitus and Alzheimer's disease. J Diabetes Res. 2020;2020:4981814.

38. Tani Y, Fujiwara T, Kondo K. Association between adverse childhood experiences and dementia in older Japanese adults. JAMA Netw Open. 2020;3(2):e1920740. https://doi.org/10.1001/jamanetworkopen.2019.20740.

39. Piirainen S, Youssef A, Song C, Kalueff AV, Landreth GE, Malm T, et al. Psychosocial stress on neuroinflammation and cognitive dysfunctions in Alzheimer's disease: the emerging role for microglia? Neurosci Biobehav Rev. 2017;77:148-64. https://doi.org/10.1016/j.neubiorev.2017.01.046.

40. Burke SL, O'Driscoll J, Alcide A, Li T. Moderating risk of Alzheimer's disease through the use of anxiolytic agents. Int J Geriatric Psychiatry. 2017;32(12): 1312-21. https://doi.org/10.1002/gps.4614.
41. van Dalen JW, van Wanrooij LL, Moll van Charante EP, Brayne C, van Gool WA, Richard E. Association of Apathy with Risk of incident dementia: a systematic review and meta-analysis. JAMA Psychiatry. 2018;75(10):1012-21. https://doi.org/10.1001/jamapsychiatry.2018.1877.

42. Mah L, Binns MA, Steffens DC. Anxiety symptoms in amnestic mild cognitive impairment are associated with medial temporal atrophy and predict conversion to Alzheimer disease. Am J Geriatric Psychiatry. 2015; 23(5):466-76. https://doi.org/10.1016/j.jagp.2014.10.005.

43. Sims R, Hill M, Williams J. The multiplex model of the genetics of Alzheimer's disease. Nat Neurosci. 2020;23(3):311-22. https://doi.org/10.103 8/s41593-020-0599-5.

44. Meng X, D'Arcy C. Education and dementia in the context of the cognitive reserve hypothesis: a systematic review with meta-analyses and qualitative analyses. PLoS One. 2012;7(6):e38268. https://doi.org/10.1371/journal.pone. 0038268.

45. Zhang $Q, X u F$, Wang L, Zhang WD, Sun CQ, Deng HW. Detecting potential causal relationship between multiple risk factors and Alzheimer's disease using multivariable Mendelian randomization. Aging. 2020;12(21):21747-57. https://doi.org/10.18632/aging.103983.

46. Fayosse A, Nguyen DP, Dugravot A, Dumurgier J, Tabak AG, Kivimäki M, et al. Risk prediction models for dementia: role of age and cardiometabolic risk factors. BMC Med. 2020;18(1):107. https://doi.org/10.1186/s12916-020-01 578-x.

\section{Publisher's Note}

Springer Nature remains neutral with regard to jurisdictional claims in published maps and institutional affiliations.

\section{Ready to submit your research? Choose BMC and benefit from:}

- fast, convenient online submission

- thorough peer review by experienced researchers in your field

- rapid publication on acceptance

- support for research data, including large and complex data types

- gold Open Access which fosters wider collaboration and increased citations

- maximum visibility for your research: over $100 \mathrm{M}$ website views per year

At BMC, research is always in progress.

Learn more biomedcentral.com/submissions 